\title{
Advances in mathematical modelling of biofilm structures
}

\author{
C. S. Laspidou ${ }^{1}$, A. Kungolos ${ }^{2} \&$ P. Samaras ${ }^{3}$ \\ ${ }^{1}$ Hydromechanics and Environmental Engineering Laboratory, \\ Department of Civil Engineering, University of Thessaly, Greece \\ ${ }^{2}$ Department of Housing and Regional Development, \\ University of Thessaly, Greece \\ ${ }^{3}$ Technological Institute of Western Macedonia, \\ Department of Pollution Control Technologies, Greece
}

\begin{abstract}
Biofilms are found everywhere in natural waters forming complex microbial communities, playing an important role in ecosystem processes in streams and lakes and making their characterization a major research question. Biofilm models are commonly used as simulation tools in engineering applications and as research tools to identify and fill gaps in our knowledge of biofilm processes. Recently, mathematical biofilm models are becoming more complex and use advanced computational tools to predict the new structure formed under a set of given conditions, as well as the microbial species that comprise the biofilm structure. Moreover, advanced biofilm models incorporate the hydrodynamics of the fluid surrounding the growing biofilm and translate it into forces that act on the biofilm and cause detachment. Thus, they enhance their predictive capability over traditional biofilm models and enable research scientists and engineers to evaluate the relevance of biofilm heterogeneities to their function. An overview of the features of the unified multi-component cellular automaton (UMCCA) model is presented. The UMCCA model describes quantitatively the complexity of biofilms for all biofilm components: active bacteria, inert biomass, and extracellular polymeric substances (EPS). It also includes original donor substrate, two types of soluble microbial products (SMP), and oxygen. The UMCCA model captures all trends observed experimentally regarding biofilm density by employing the novel idea of biofilm consolidation, according to which the biofilm packs itself to a higher density over time. The UMCCA model can also be used to describe biofilm mechanical properties variable in time and space, making it possible to predict where it is likely to fail, or detach.

Keywords: biofilm modelling, extracellular polymeric substances, EPS, biofilm density, inert biomass, active biomass, soluble microbial products, SMP.
\end{abstract}




\section{Introduction}

Biofilms are ubiquitous in nature and play a critical role in various biological processes in streams and lakes. Factors responsible for the abundance and growth of periphyton a natural biofilm found in streams and lakes, have been studied previously Hepinstall and Fuller [1], Rier and Stevenson [2], Sobczak [3]. Such factors that may affect microbial communities in streams and lakes are dissolved organic matter and inorganic nutrients. Biofilms are layerlike aggregations of microorganisms and their extracellular polymeric substances (EPS) attached to a solid surface. Some biofilms are viewed as "good" and we try to promote their accumulation. Examples are those exploited in fixed-film processes used to treat contaminated water, wastewater and air, or those that attach to stream beds and aquatic vegetation leading to self-purification of water bodies, and those responsible for engineered or intrinsic bioremediation of contaminated groundwater. Other biofilms are viewed as "bad," and we try to remove or prevent them. Bad biofilms include those that foul ship hulls, thereby increasing friction loss and corrosion and those that accumulate in pipes in water distribution systems possibly carrying pathogens or corroding them. Thus, microbial biofilms can be of great benefit but they can also be a nuisance. Humans may want to control or enhance their presence, both in technical and natural systems. Therefore, it is extremely important to understand the structure and function of biofilm communities as well as the mechanisms regulating biofilm processes.

Biofilms are highly heterogeneous and diverse. They could be thick or thin, "fluffy" or dense, forming a solid mat or having finger-like protrusions in the overlying fluid and having clusters or streamers of biomass intermingled with open channels. They could include several different types of microbial species, such as heterotrophs and autotrophs. Even when biofilms do not include different microbial types, they are still heterogeneous since bacteria always produce EPS and "inert" or dead biomass Laspidou and Rittmann [4]. Biofilm research faces invariably the challenge of understanding complex relationships between physical, chemical and biological processes occurring at very different spatial and temporal scales. The best tool available for integrating the plethora of experimental observations in a rational environment is mathematical modeling. Mathematical models are no more than a simplified representation of reality consisting of sets of equations and algorithms containing the information needed to simulate a system. Models can lead to a deeper understanding of the underlying principles and to the potential of making predictions, so they are valuable computational tools.

Microbial biofilms have been simulated by mathematical models for the last three decades Noguera et al [5]. The initial models described biofilms as uniform steady-state films containing a single type of organism, governed exclusively by one-dimensional mass transport and biochemical transformations Rittmann and McCarty [6]. Later, layered dynamic models were developed Wanner and Gujer [7], which included multi-species interactions within the biofilm, but were not able to represent the characteristic structural heterogeneity 
that has been recently elucidated through experimental observations. The development of advanced microscopy techniques like Confocal Scanning Laser Microscopy (CSLM) has allowed researchers to really see what biofilms look like; thus, it became obvious that they do not grow in flat layers, but have channels and pores, and mushroom-like or tulip-like protrusions in the fluid, depending on the conditions the biofilm is grown under (Figure 1). It was therefore shown that the models that wanted the biofilms growing in layers were not really capturing the structural heterogeneity of biofilms; this realization motivated an explosion in the development of mathematical models during the past decade. This explosion was also motivated by the advances in computing capabilities that made it possible to perform the massive calculations that the new models needed, in a short period of time.

\section{Objectives of biofilm modeling}

During biofilm development, a large number of phenomena occur simultaneously and interact over a wide range of length and time scales. Nutrients are being taken up and converted and the biofilm expands as a result of bacterial growth and production of EPS. Chemical species are continuously transported to and from the biofilm system by physical processes such as molecular diffusion and convection. Fluid flow influences biofilm growth in two ways: first, by determining the concentrations of available substrates and products and second, by shearing the biofilm surface with a detachment process Rittmann and Laspidou [8]. In the case of multi-species systems, microorganisms of different species interact in complex relationships of competition or cooperation. All these phenomena create a dynamic picture of the biofilm structure. Mathematical models can prove useful because they allow testing of hypotheses and, in addition, can direct experimental efforts to complex regions of operation that need to be further investigated. In addition, biofilm models are frequently used by practicing engineers as a simulation tool to analyze the performance of biofilm processes. The models provide engineers with the means to evaluate the significance of several parameters, allowing them to search for explanations of performance problems. Thus, it is possible to formulate hypothetical modifications in operation and to simulate process behavior in response to operational changes before full-scale implementation.

In relation to research applications, mathematical models can provide useful insights into the evaluation of biofilm parameters, especially when used in combination with experimental results. As for practical engineering applications, the current objectives of biofilm modelling include biofilm engineering, real-time control and applications in education. Therefore, models can help engineers gain an insight into the interactions between the processes involved in biofilm formation so that it would be possible to design the biofilm structure and its function. For example, the environmental conditions can be manipulated to generate dense biofilm structures that will be easily separated from a liquid phase (as in a fluidized bed reactor), or multi-layered biofilms that would block corrosion of ship hulls, or rough biofilm structures with high capacity for removal of particulate material. 


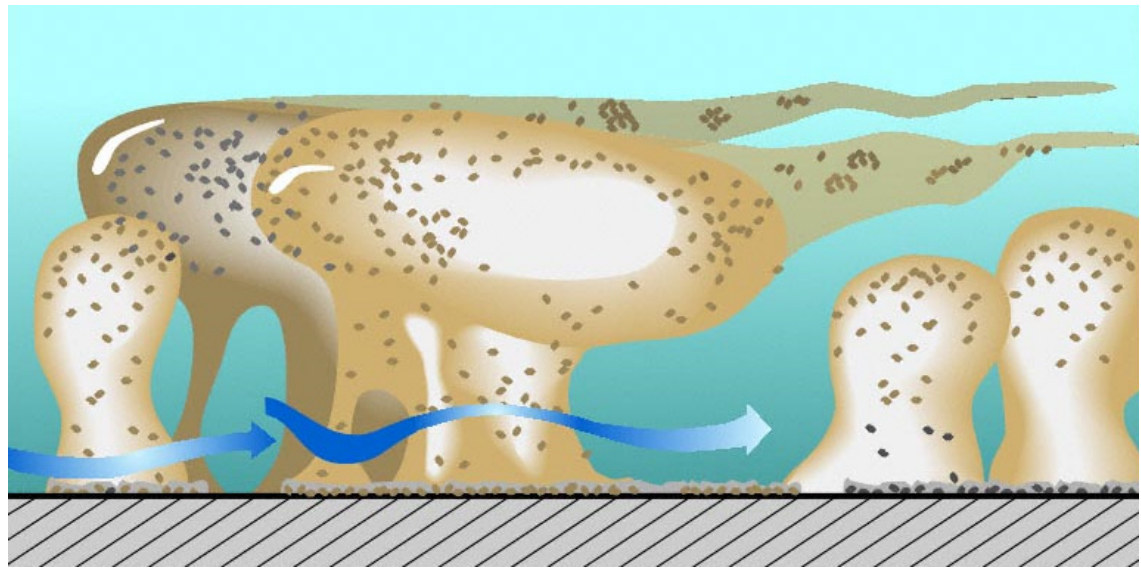

Figure 1: Conceptual illustration of the heterogeneity of biofilm structure, showing bacterial clusters, streamers, and water channels (adapted from a picture by the Center for Biofilm Engineering, Montana State University-Bozeman).

But why is it important to know the biofilm structure in the first place? The structure of biofilms plays a very important role in their activity. Together with the liquid flow, the shape of the biofilm surface influences the transport of all solutes from the bulk liquid to the microbial cells where the bioconversions occur. The structure, in turn, is itself defined by the biofilm activity. Bacterial cell growth and division, as well as EPS production and secretion, together with external factors such as shear forces defined by hydrodynamics and other mechanical forces shape the biofilm structure Picioreanu et al [9]. Depending on these factors, the biofilm develops at a variable density, i.e. it is either loose and "fluffy" or dense and compact. Naturally, biofilm density plays a critical role in biofilm detachment. Therefore, in a cyclical almost way, the hydrodynamics apply forces on the biofilm and cause detachment thus forming its surface, which in turn plays a role in substrate transport, defines biofilm activity and growth, which changes the biofilm surface and once again plays a role in hydrodynamics (Figure 2).

\section{The UMCCA model}

A sophisticated mechanistic model of biofilm structures is the Unified MultiComponent Cellular Automaton (UMCCA) model, which predicts quantitatively all biofilm components: active bacteria, inert (or dead) biomass produced by death and decay, and EPS. An overview of the model is presented in Laspidou and Rittmann [10]. This model is the biofilm adaptation of the multi-component mathematical model that quantifies the previously developed unified theory that reconciles the apparently disparate findings about active and inert biomass, EPS and other microbial products Laspidou and Rittmann $[4,11]$. The unified theory 
provides a set of mathematical equations that describe the development of all solid and soluble species in the biofilm. The UMCCA model represents a growing biofilm using a cellular automaton (CA) approach in which the biofilm grows in a two-dimensional domain of compartments.

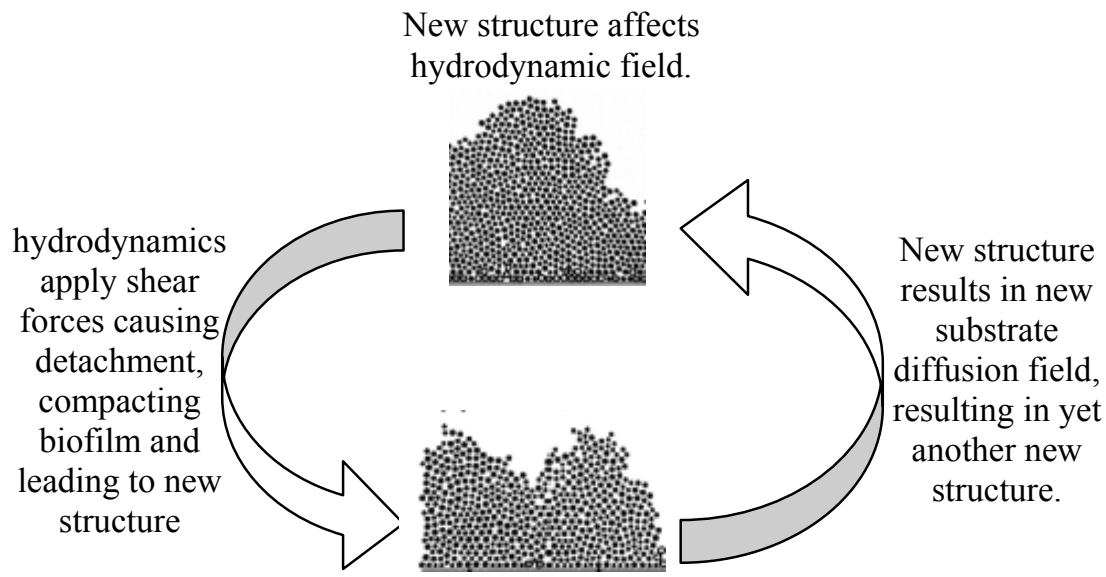

How much biofilm detachment occurs

depends on biofilm density.

Figure 2: Hydrodynamics and substrate diffusion interact in forming the biofilm structure.

One of the unique features of the UMCCA model is that it can predict a variable biofilm density throughout the biofilm column. This is important as experiments show that the biofilm that is closest to the substratum has the highest density, while the top layers remain "fluffy" and porous, with the bottom of the biofilm being as much as 5 to 10 times denser than the top Bishop et al [12]. Biofilm porosity follows opposite trends, suggesting that biofilm accumulates near the substratum with a closer packing arrangement than at the top. In order for UMCCA to capture these density differences, it employs the theory of consolidation, an idea borrowed from the fields of geotechnical engineering Rittmann and Laspidou [13, 14]. According to this theory, when pressure is applied to a bed of irregularly sized solids, the solids gradually pack to a higher density, or consolidate. The solids do this to minimize porosity and achieve a lower energy state. When the applied pressure is combined with vibration consolidation is accelerated. Because a biofilm is a matrix of particles, UMCCA includes equations that allow it to consolidate, or gradually pack to a higher density, due to the differential forces, such as friction, which have developed as a result of the fluid flowing over it. Thus, the bottom biofilm layers, those closest to the substratum undergo consolidation for the longest time; therefore, the bottom layers should have the highest densities and the lowest porosities, which is what Bishop et al [12] observed in their experiments. 
The UMCCA model is a hybrid differential and discrete cellular automaton (CA) model; thus, solutes are represented in a continuous field by reactiondiffusion mass balances, while solids are mapped in a discrete cell-by-cell fashion that uses a CA algorithm. The CA algorithm is a set of rules that determines where to place the newly-formed biomass, how to expand the biofilm front by "pushing" neighbouring cells when new biomass grows in. The model includes seven major variables: active biomass, EPS and inert biomass, as well as original donor substrate, two kinds of soluble products and oxygen. The model's general objective is to describe quantitatively the heterogeneity of biofilms.

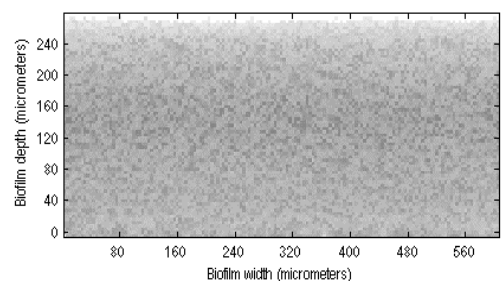

(a)

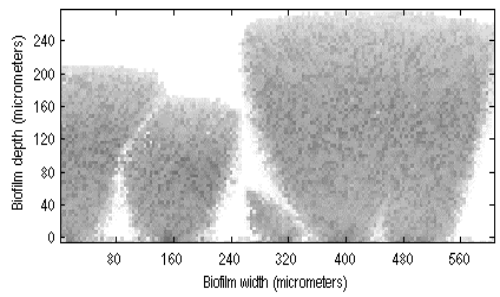

(c)

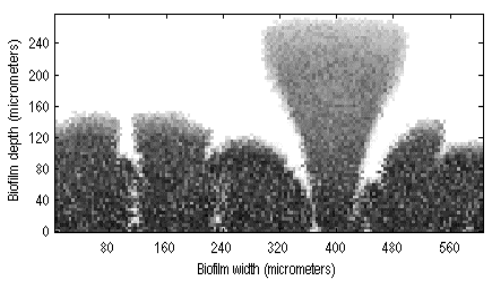

(b)

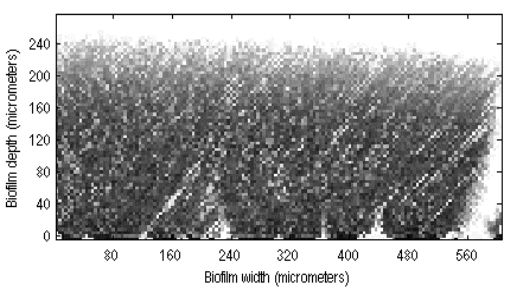

(d)

Figure 3: Sample UMCCA model outputs of composite density. (a) Standard conditions (24.5 days of simulated time), (b) low substrate concentration (221 days), (c) high detachment rate (30 days) and (d) low oxygen conditions (195 days).

Figure 3 shows some sample model outputs of composite density. These are presented in a shading format, with a shade of grey being associated with a composite density: a black compartment represents a biofilm location that is filled completely $(100 \%)$ with a combination of solid species, while a white compartment represents an empty compartment. Figure 3 shows that different conditions produce different physical structures. Biofilms may grow in "mat" formations (Figure 3(a)), in "mushrooms" (Figures 3(b) and (c)) and in mat formations with "holes" close to the substratum (Figure 3(d)). Figure 3(b) and (d) show biofilms with much higher densities at the bottom, while Figures 3(a) and (c) show lower and more uniform densities throughout the column of the 
biofilm. But why are the biofilm structures in Figure 3 so different so different with respect to density and heterogeneity? Before this question is answered, an analysis of the composite density graph in Figure 3(a) is presented (shown again in Figure 4(d)).

Figure 4 shows a breakdown of the individual solid species that comprise the biofilm density (active biomass, EPS and residual inert biomass) for the composite density in Figure 3(a), which corresponds to a simulation of biofilm after 24.5 days. Figure 4(a) tells us that active biomass is close to zero at the bottom of the biofilm, since it had enough time to decay almost completely to residual inert biomass, which is what is shown in Figure 4(b). Active biomass is also at a low density at the top of the biofilm; here, the reason is that the biofilm in these rows is young and has had no time to fill in all the space and consolidate. Hence, the density of active biomass peaks at about one-quarter of the depth of the biofilm, where substantial synthesis has had time to occur, but decay is not yet dominant. Residual biomass, shown in Figure 4(b) is produced only after active biomass is synthesized and has had enough time to decay. Figure 4(c) shows that EPS follows the active-biomass profile. The total composite density, Figure 4(d), is a composite of the three biomass component. Its maximum is close to the middle of the biofilm. The composite density for this case is not especially high, compared to Figures 3(b) and (d), since the age of this biofilm is modest, only 24.5 days.

The graph of composite density in Figure 3(b) is very different from that in Figure 3(a), since it is a result of a run with lower substrate concentration. The slower biofilm growth rate, due to the lower substrate concentration, required 221 days of simulated time. At the bottom of the biofilm, substrate is nearly zero and active biomass has had a long time to decay almost totally to inert biomass. In Figure 3(b), it appears that a cluster protrudes higher than the rest of the biofilm; this happens because, once a cluster manages to protrude, it is exposed to a higher substrate concentration than the other clusters are, it gets a growthrate advantage and then it keeps growing faster than the rest of the biofilm. Figure 3(c) shows that a high-detachment-rate run took 30 days to fill the domain. The run was relatively short, because the composite density is lower overall, which means that less biomass is needed to fill the domain. The biofilm has a lower proportion of inert biomass (the highest-density solid species), because the loss of active biomass is shifted from decay to detachment. Figure 3(d) is a low-oxygen run that terminated in 195 days. In the absence of oxygen, active biomass growth ceases, while decay (not an oxygen-dependent process) proceeds. As a result, most of the biofilm is almost completely inert, with "holes" at its bottom that were unable to fill up with biomass owing to the lack of oxygen.

\section{UMCCA and mechanical properties: the next phase}

A new feature that was added to the UMCCA model is the ability to link a physical property-like composite density or the densities of active biomass, EPS, and inerts - to the mechanical properties of the biofilm, namely the 
Young's modulus E and Poisson ratio $v$ (reference to the meaning of these new terms). In this way, we can use the UMCCA model to predict mechanical properties for the biofilm, and the mechanical properties can vary in time and in space. The significance of this feature is that we may gain insight into the issue of biofilm detachment, which can be viewed as a mechanical "failure" of the biofilm structure. Physical causes of detachment include forces acting on the biofilm structure itself, which most probably come from the fluid surrounding and moving around the biofilm and exerting shear stresses on it. Since the biofilm is heterogeneous, it is reasonable to assume that mechanical properties are not uniform either; thus, an analysis that predicts the mechanical properties throughout the biofilm is what is necessary to understand when, where, and why biofilm detachment occurs.

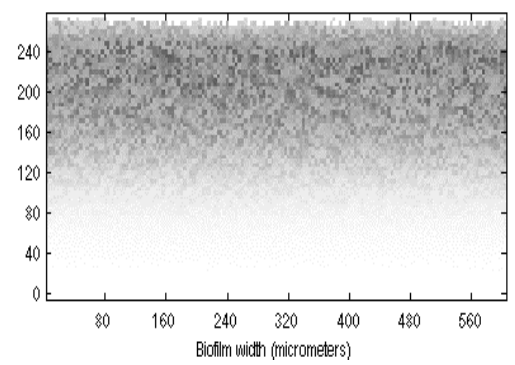

(a)

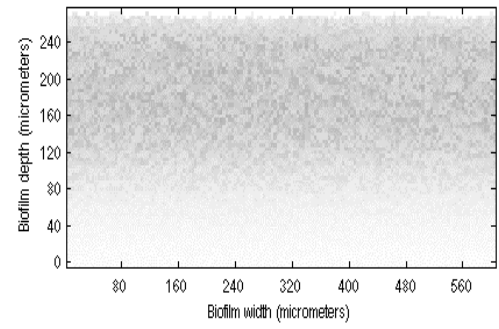

(d)

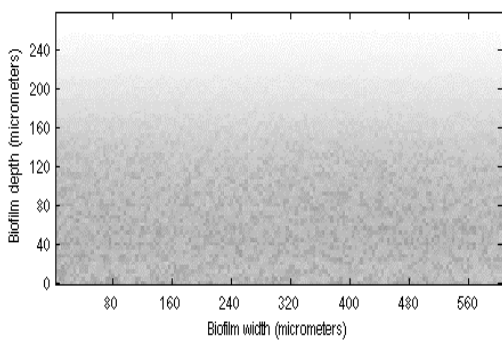

(b)

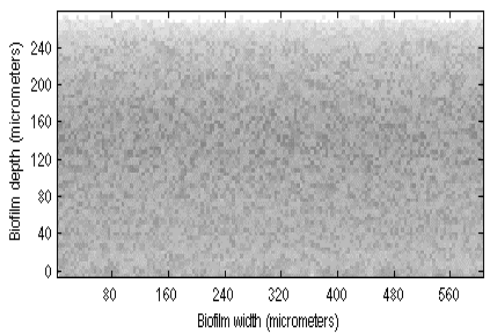

(d)

Figure 4: UMCCA model outputs of (a) active biomass (b) residual dead biomass (c) EPS and (d) the corresponding graph of composite density for the simulation also shown in Figure 3(a).

Once the variable mechanical properties of the biofilm from the UMCCA outputs are computed, they can be imported to a finite-element software used in structural mechanics, such as ABAQUS. When the forces acting on the biofilm and boundary conditions are provided, ABAQUS can be used to show how biofilms deform and what the internal stresses in the biofilm are. Locations where failure is likely can be predicted because the internal stress at that location exceeds the biofilm's yield strength (tensile or compressive). Limited tensile 
strength data were published by Ohashi et al [15] and can be used as a comparison level for such studies. A preliminary case study motivated from the experiments in [15] is presented in Laspidou et al [16].

\section{Conclusions}

Multi-dimensional modeling of biofilm structures constitutes a valuable tool for the investigation of the biofilm's structure as a result of its activity and the diffusion - reaction coupling. An overview of a quantitative biofilm model - the UMCCA model - that generates realistic biofilm structures formed under a set of given conditions relevant to natural ecosystems is presented. The UMCCA model incorporates the substrates and hydrodynamics of the fluid surrounding the growing biofilm and simulates the growth of biofilm structures predicting the different species that comprise the biofilm. It describes quantitatively the complexity of biofilms for all biofilm components: active bacteria, inert biomass, and extracellular polymeric substances (EPS). It includes original donor substrate, such as dissolved organic matter in a stream or lake, two types of soluble microbial products (SMP), and oxygen. The UMCCA model also employs the novel idea of biofilm consolidation, according to which the biofilm packs itself to a higher density over time. It can also be used to describe biofilm mechanical properties variable in time and space, making it possible to predict where it is likely to fail, or detach.

\section{References}

[1] Hepinstall, J.A. \& Fuller, R.L., Periphyton reactions to different light and nutrient levels and the response of bacteria to these manipulations. Arch. Hydrobiol., 131, pp. 161-173, 1994.

[2] Rier, S.T. \& Stevenson, R.J., Effects of light, dissolved organic carbon, and inorganic nutrients on the relationship between algae and heterotrophic bacteria in stream periphyton. Hydrobiologia, 489, pp. 179184, 2002.

[3] Sobczak, W. Epilithic bacterial responses to variation in algal biomass and labile dissolved organic carbon during biofilm colonization. J. Norht Am. Benthol. Soc., 15, pp. 143-154, 1996.

[4] Laspidou, C.S. \& Rittmann, B.E., A unified theory for extracellular polymeric substances, soluble microbial products, and active and inert biomass. Water Research, 36, pp. 2711-2720, 2002.

[5] Noguera, D.R., Okabe, S. \& Picioreanu, C., Biofilm Modeling: Present Status and Future Directions. Water Science and Technology, 39(7), pp. 273-278, 1999.

[6] Rittmann, B.E. \& McCarty, P.L. Model of Steady-State Biofilm Kinetics Biotechnology and Bioengineering, 22, pp. 2343-2357, 1980.

[7] Wanner, O \& Gujer, W., A multispecies biofilm model Biotechnology and Bioengineering, 28, pp. 314-328, 1986. 
[8] Rittmann, B. E. \& Laspidou, C. S. Biofilm Detachment. The Encyclopedia of Environmental Microbiology, ed. G. Bitton, John Wiley \& Sons, Inc., New York, 2001.

[9] Picioreanu, C., Xavier, J.B. van Loosdrecht, M.C.M., Advances in mathematical modeling of biofilm structure, Biofilms, 1(4), pp. 301-313, 2004.

[10] Laspidou, C.S. \& Rittmann, B.E., Modeling biofilm complexity by including active and inert biomass and EPS. Biofilms, 1(4), pp. 285-291, 2004.

[11] Laspidou, C.S. \& Rittmann, B.E., Non-steady state modeling of extracellular polymeric substances, soluble microbial products, and active and inert biomass, Water Research 36, pp. 1983-1992, 2002.

[12] Bishop, P.L., Zhang, T.C. Fu, Y.-C., Effects of biofilm structure, microbial distributions and mass transport on biodegradation processes, Water Science and Technology, 31(1), pp.143-152, 1995.

[13] Laspidou, C.S \& Rittmann, B.E. Modeling the development of biofilm density including active bacteria, inert biomass, and extracellular polymeric substances, Water Research 38(14-15), pp. 3349-3361, 2004.

[14] Laspidou, C.S. \& Rittmann, B.E., Evaluating trends in biofilm density using the UMCCA model, Water Research, 38(14-15), pp. 3362-3372, 2004.

[15] Ohashi, A., Koyama, T., Syutsubo, K., Harada, H., A novel method for evaluation of biofilm tensile strength resisting to erosion. Water Science and Technology, 39(7), pp. 261-268, 1999.

[16] Laspidou, C.S., Rittmann, B. E. Karamanos, S.A. Finite-element modeling to expand the UMCCA model to describe biofilm mechanical strength, Water Science and Technology, 52(7), pp. 161-166, 2005. 